\title{
The Relevance of Synergy Between Forensic Pathologist and Toxicologist in Medico-Legal Autopsies
}

\author{
Lorenzo Desinan $^{1}$, Antonio Colatutto ${ }^{2, *}$ and Pierguido Sala ${ }^{2}$ \\ ${ }^{I}$ Department of Experimental and Clinical Medicine/Legal Medicine, University of Udine Medical School, Udine \\ ${ }^{2}$ Department of Laboratory Medicine, Clinical Pathology, Hospital S. Maria della Misericordia, Udine
}

\begin{abstract}
In forensic toxicology the main relevant scope of toxicologist is to answer these following questions: which kind of drug is involved? Is the analytical result related to death? Which is the supposed lethal dose? Despite the technological improvement in laboratory medicine, the main questions are apparently unresolved, because the availability of new laboratory instrumentation has sometimes increased the complexity of the medical examiner task. In post mortem toxicology the medical examiner's and the toxicologist's role is constantly challenged by analytical, methodological, circumstantial problems, and only the single professional experience can help to find which drug is involved.

In our experience the only way to avoid, or limit, mistakes is to follow strictly a Search Strategy, which combines single professional experience, circumstantial data and a correct and a punctual manner of sampling from corpses. The most complete and proper tissue sampling from autopsy is mandatory for the whole diagnostic process. This approach in synergy with the pathologist and toxicologist dialogue can help the medical examiner to avoid mistakes and positively infer the whole diagnostic process.
\end{abstract}

Keywords: Post mortem toxicology, gas chromatography, high performance liquid chromatography, xenobiotic, autopsy, alternative matrices.

\section{INTRODUCTION}

According to Recommendation No. R (99) 3 of the Committee of Ministers to Member States on the Harmonisation of Medico-Legal Autopsies, autopsies should be carried out in every suspected unnatural death, even where there is a delay between causative events and death (Table 1). In particular, autopsies should be performed in homicide or suspected homicide, in sudden, unexpected death, including sudden infant death, in violation of human rights such as suspicion of torture or any other form of ill treatment, in suicide or suspected suicide, in suspected medical malpractice, in accidents caused by occupational, domestic, or transport, in occupational disease, in technological or environmental disasters, in death in custody or death associated with police or military activities and in unidentified or skeletonised bodies [1].

Medico-legal autopsies performed by forensic pathologist in cases of poisoning can be amongst the most difficult of problems faced, not in the technical procedure of the examination, but in the final evaluation of all available information [2].

Post mortem toxicology represents a special field of forensic toxicology with a rather small number of scientists (mostly forensic scientist) worldwide. Since substrates which are examined in post-mortem toxicology are often seriously

*Address correspondence to this author at the Department of Laboratory Medicine, Clinical Pathology, Hospital S. Maria della Misericordia, Udine; Tel: 00390432 552406; Fax: 00390432552402 ;

E-mail: colatutto.antonio@aoud.sanita.fvg.it influenced by post-mortem degradation, redistribution, matrix, temperature, etc. the interpretation of the examination results becomes difficult. Interpretation of post mortal toxicological results is always based on own experiences as well as on comparisons with literature.

The circumstances of the case, the previous diseases history and autopsy findings are essential for the interpretation, too. Defining the cause of death-specially the question if the toxicological findings alone are the cause of death or if they are one of the causes of death among others can be simplified by taking into consideration the graduation of findings regarding their importance as cause of death and the evaluation of the dying type [3].

Table 1. Recommendation No. $R$ (99) of the Committee of Ministers to Members States on Harmoni-sation of Medico Legal Autopsies

\begin{tabular}{|l|}
\hline Homicide \\
\hline \hline Sudden death \\
\hline Violation of human rights \\
\hline Suicide \\
\hline Suspected malpractice \\
\hline Accidents \\
\hline Occupational diseases \\
\hline Enviromental disasters \\
\hline Death in custody \\
\hline Unidentified bodies \\
\hline
\end{tabular}


For the reasons mentioned above we think that a synergic approach between forensic pathologist and toxicologist is specially relevant.

\section{THE PATHOLOGIST'S POINT OF VIEW}

\section{The Procedure for a Medico-Legal Autopsy}

A medico-legal autopsy has wider aims than those of a clinical autopsy and includes: to identity of the body; to estimate the time of death; to identify and document the nature and number of injuries; to identify the presence of any natural disease; to interpret the significance and effect of the possible natural disease; to identify the presence of poisons; and to interpret the effect of any medical or surgical treatment (Table 2) [4].

Table 2. Aims of Medico-Legal Autopsy

\begin{tabular}{|l|}
\hline Identification of the body \\
\hline \hline Estimation of the time of death \\
\hline Identification of nature and number of injuries \\
\hline Identification of natural diseases \\
\hline Interpretation of the effects of natural disease \\
\hline Identification of presence of poison \\
\hline Interpretation of medical and surgical treatments \\
\hline
\end{tabular}

Before performing a medico-legal autopsy it is necessary to reflect on the instructions of the legal authority responsible on the investigation of the case and to know the results of previous activities (both clinical and investigative).

Clinical notes, in cases of poisoning, inform in particular about previous assumption of substances and can also describe the symptoms presented immediately before death. In this case the analytical search not only directs towards the general diagnosis of poisoning but also towards the specific type of substance implicated.

Investigative notes with the photographs of the body and of the scene and surrounding add additional useful information on circumstances of death, and crime can sometimes rapidly be excluded in favor of accident, suicide or even natural causes.

Of particular relevance for the forensic toxicologist are the findings of paraphernalia, drugs and residues of substances on the scene.

So a first phase of interaction between forensic pathologist and toxicologist should be before performing the autopsy to decide how to proceed and the possible problems to be solved.

However the technical procedure of the autopsy in suspected poisons is not substantially different from standard one, major difficulties arising in the final evaluation of all available information.

\section{External Examination}

In any case it is mandatory that the pathologists performs a systematically external examination, because the examination performed in life is often poorly documented and full of mistakes, for example lacerations described as incised wounds or natural skin lesions are described as bruises [5].

In cases of poisoning it is relevant, at external examination, the presence of:

- $\quad$ damage of peripheral veins by the repeated injection of drugs most commonly in the arms, hands and legs (sometimes in the groin or neck);

- cherry-pink post-mortem hypostasis of carbon monoxide poisoning;

- $\quad$ overflow marks around the corners of the mouth and driblets that run down the chin, neck, chest by acid or alkali corrosive agents;

- $\quad$ the rare ulceration of nasal septum in long-term nasal abuse of cocaine.

\section{Internal Examination}

After the pathologist has performed a detailed external examination, the body may be opened and eviscerated to permit a detailed examination of the viscera.

It is a pity that, in general, no specific signs of intoxication are present at the examination of various organs, with the exception of cherry-pink color also of internal organs in monoxide intoxication, or the morphological equivalent of action of corrosive agents in aero-digestive tract, or the findings of residuals of the drugs assumed within the stomach content.

The main significance of internal examination of various organs is the documentation of the presence of the various pathologies, for the final discussion of the case, when the conclusions, in synergy between forensic pathologist and toxicologist, are about the possibility that the poison is alone able to explain the death, or it has had a causative action with the pathologies present, or it has not had a causal role even if present in significant quantities.

Particularly controversial are the cases in which no toxic substances are present, even if there was a documentation of that, because catabolism has made its destructive action.

\section{Suspicion of Intoxication}

According to Recommendation No. R (99) 3 of the Committee of Ministers to Member States on the Harmonisation of Medico-Legal Autopsies, where anatomical findings do not reveal a cause of death and/or there is vague suspicion of poisoning, basic sampling should include in addition to peripheral blood, urine, and stomach contents (to be done in all autopsies), and if specific suspicion arises, sampling should be group-related as follows [1]:

hypnotics, sedatives, psycho-active drugs, cardioactive drugs and analgesics, pesticides: as aforementioned under 1;

- drugs of abuse: as aforementioned under 1 and additionally cerebrospinal fluid, brain tissue, injection marks, hairs;

- $\quad$ volatile fat-soluble substances such as fire accelerants and solvents: as aforementioned under 1 and 
additionally: blood from left ventricle, brain tissue, subcutaneous fat tissue, lung tissue, clothing;

- nutritional intoxication: as aforementioned under 1 and in addition: intestinal contents, if possible taken from 3 different sites;

- $\quad$ suspicion of chronic intoxication (heavy metals, drugs, pesticides) as aforementioned under 1 and in addition: hairs (tufts), bones, fat tissue, intestinal contents.

On the above scheme the various laboratories elaborate their own personal protocols.

\section{Sampling}

Of main importance to obtain good results is the quality of sampling of the various biological matrices that are to be collected in adequate quantities (consider the necessary amount, in case of controversies, to repeat the analysis a second time) [2].

Sometimes it happens that the desired set of sampling cannot be obtained for the state of conservation of the body, and in those cases an additional trouble derive from the necessity of using suboptimal materials.

Freezing is the conservation method for the material collected.

According to the personal protocols in use the following points can be underlined:

1. Urine is best collected by puncture with a syringe of the bladder after the opening of the abdominal cavity. In the case of a bladder empty of urine we recommend to wash the bladder wall to collect all the residual urine. Urine is particularly useful for screening investigations. If the subject was recently subjected to vesical catheterisation, the fact has to be communicated to the toxicologist for the presence of anaesthetics. Urine is considered the best matrix for screening analyses.

2. Blood. Even if some authors are against the use of central blood, we recommend the collection of both peripheral and central blood. Peripheral blood should be collected at the beginning of autopsy to avoid contamination, and obtained from the femoral artery or vein by percutaneous puncture with a syringe. Central blood is collected from cardiac cavities. Blood outside the vessels (from the paracolic gutter for example) is not suitable for analyses, and should be considered only if no others fluids are available, for example in the case of a second autopsy. Tubes containing a fluoride preservative should be used for alcohol, cyanide or cocaine metabolite analyses.

3. Bile is aspirated from the gallbladder before abdominal evisceration. In case of cholecystectomy useful quantities of bile can be collected from common bile duct using a syringe.

In cases of paracetamol overdose significant concentration of the drug can be present in the bile when blood shows very low levels.
4. Vitreous humour is obtained by needle puncture of the eyeball directed from lateral-anterior position to the centre of the organ. We are in favor of a systematic use of this material, that in case of difficulties to collect suitable amounts of blood can be a valuable alternative.

5. Hairs. They should be collected at the beginning of the autopsy to avoid contamination. The information given from this material concerns the previous toxicological history of the subject.

6. Solid organs must be collected fresh, without using fixatives: particularly valuable are $\underline{\text { Liver }}$ (the main site of metabolic processes), Kidney (for his emunctory function) and Heart and Brain (because of the toxic substances having main action on these organs).

7. Stomach content samples or the collection of the entire material is mandatory as a routine procedure: in some case it is possible to recognize some drugs not yet digested, and in every case relevant information is given about recent assumption of substances using this way.

8. Other possibilities to be considered is collection of samples from cerebrospinal fluid, adipose tissue, lung, small bowel content and faeces.

\section{THE TOXICOLOGIST'S POINT OF VIEW}

Even tough Forensic Toxicology could be not defined generally speaking a recent discipline itself, only in recent years the technological progress has really improved the reliability of the whole analytical process. Despite the paramount progress in technology the clinical dilemma is the same: which drug or toxicant did cause the death? Paradoxically to answer this question is more difficult because the improvement of our knowledge, and for the improved accuracy and sensibility of so called innovative toxicological tests.

While the main scope of clinical pharmacology is to study the direct relationship between the dose of the drug and its pharmacological effect, in post mortem and forensic toxicology the knowledge of pharmacokinetic and pharmacodynamics parameters is scarcely useful to understand the cause of death. Nevertheless the consistent progress of new laboratory technology, that has improved both sensitivity and specificity of toxicological analysis, has lead the forensic toxicologist to get "powerful weapons" to establish the punctual concentration of detected drugs. However it must be stressed that every experienced forensic toxicologist should be aware that data extrapolation could determine erroneous conclusions, so the improvement of diagnostic performance comes from the possibility to study alternative matrices too. In this latter contest it should also be stressed that the most difficult task is to define toxic or lethal dose of a drug or toxicant.

Nevertheless the increased load of analysis has lead the pharmacologist and the toxicologist to explore a rational approach to the whole diagnostic process, and the only way to achieve this goal is to follow a shared path as to secure the analytical global quality. 
A careful check of the quality of analytical results is mandatory in forensic toxicology, and every analytical laboratory has a standard set of procedures that provide information to laboratory staff. The reliability of data produced is strictly related to Quality Assurance Procedures which are clearly defined by appropriate guide lines provided by scientific societies. The corner-stone for obtaining reliable data is firstly to provide a reliable "Custody Chain" so every specimen can be univocally identified and not corrupted.

\section{Screening Versus Confirmation Methods and Laboratory Facilities}

It is well known in routine toxicological practice that every sample could be screened by fast immunochemical or immunoenzymatic method, but positive samples must be processed by chromatography associated with mass spectrometry method (either High Performance Liquid Chromatography or Gas Chromatography). Actually screening methods are fast, not too expensive, easy to perform, and fit to not extremely trained laboratory staff. Chromatographic methods either gas or HPLC need some facilities, whose characteristics are not ever available, specially in non specialized laboratories. Because the comprehensive analysis of toxicology sample needs a rational approach for the challenging tasks requested, recent develops in chemical and analytical chemistry have led the forensic toxicologists to explore the automatic screening in postmortem toxicology. This progress arises from the improved fully automated immunochemical and immunometric laboratory technology.

In this complex field the expected progress cannot solve every kind of problem, because the toxicologist is looking for unknown xenobiotic and only a limited number of diagnostic kit are available. Every commercial kit is fit for diagnostics in living people, but toxicologist's challenge is to adapt commercial kits to a particular kind of samples as decomposed bodies, body fluids and tissues.

Any laboratory has to adapt and validate its own methods, taking in account of reliability and plausibility of results. Every positive test must be confirmed by chromatographic (gas chromatographic or High performance liquid chromatographic), but only a limited number of mass spectra of drugs are available; there is also a limited number of deuterated standards available, and this has moved forensic toxicologists to an extensive study to adapt drugs and toxicants to be processed.

Traditionally the samples routinely utilized for toxicological analysis have included femoral or central cardiac blood, urine, bile, gastric content and sometimes vitreous humor. But this traditional approach could be not completely satisfying as to correctly diagnose the cause of death. Even the punctual knowledge of the drug concentrations could affect negatively the proper data interpretation, because the toxicologist should be aware of post mortem drug concentration, and of other factors as the synthesis, metabolism, diffusion from body cavities and the site and the time of sampling.

Because an important objective of forensic toxicology is the univocal identification of toxicants in biological material, and this task could be defined as a "general analysis", it is almost impossible, also for the best fitted laboratory, to get all the standards and all the mass spectra for all the possible toxicants or pesticides.

\section{BODY FLUIDS}

\section{Whole Blood (Plasma, Serum)}

Blood is the specimen most often routinely used to quantify drugs and toxicants, and generally speaking it should be kept in mind that blood is a omnicomprehensive term which includes also plasma and serum [6-9]. In post mortem samples it is scarcely possible a clean separation of red blood cells, because the composition of the sample is quite different from the sample obtained from a living person: samples could be putrified, hemolysed, clotted or partly clotted. For these reason all the "non homogeneous" composition of the sample can alter data analyses.

The blood sampling is a crucial step and, in agree with the most quoted and accepted Guide Lines, blood should be sampled exclusively from a peripheral vessel, and cardiac or so called central blood is not fit for toxicological analysis.

Even if cardiac blood is abundant, the sample is easy to perform and drug levels in cardiac blood are higher than in peripheral vessel, we should stress the real and possible sample contamination. There is a plenty of data about the different drug concentration in different sites; these differences arise from lacking drug distribution in different sites, from uneven ante mortem and post mortem drug distribution, from redistribution from "so called reservoir organs".

The best example of this condition could be inferred by alcohol post mortem changes. After death the stomach could be identified as a reservoir, and the alcohol can spread from stomach to close tissues and vessels. One of possible ways by which alcohol sheds is by leakage versus the left lung, the left liver lobe and left cardiac ventricle.

For this reason the best sample choice is to obtain the whole blood for ethanol quantification from femoral vein. Aside sampling troubles alcohol is a relatively stable compound, but this is not the condition of some drug of abuse as opiates or cocaine. There are also a lot of discrepancies about results in methamphetamine, amitriptyline analyses, and these stem from different sites of sampling, from post mortem changes due to $\mathrm{pH}$ variations and from early and late stages of putrefaction.

The uneven blood/plasma/tissue distribution of xenobiotic in living people is determined by active processes, but after death the afore mentioned conditions could alter analytical data so it is almost impossible to make a comparison from ante and post mortem levels of blood xenobiotics.

When we must interpret analytical data we should keep in mind the correct integration of clinical status, circumstances and post mortem changes, and it is imperative to state that blood samples can often provide only qualitative proof of the presence of analytes. This is specially true when the toxicologist is asked about lethal dose of toxicant or drugs, in this case the positive finding could only be a reliable proof of recent consumption. Blood can be sampled 
also when there is suspicion of poisoning by volatile compounds, and it is also appropriate to sample blood when there is carboxy hemoglobin poisoning [10-13].

\section{Urine}

The urine is historically the sample of choice, but unfortunately it is not always available and the analytical results are not related to the clinical condition ante death. Urine can be defined a reservoir itself, in which we find all xenobiotics taken before death. Urine is used as a screening specimen, and could be analyzed directly by immunometric or immunochemical automated instrumentation, so the toxicologist has quick view of possible foreign substances.

However every experienced toxicologist is well aware of no correlation between urine drug concentration and clinical effects: because a negative urine result could also imply that the xenobiotic has not been absorbed! Since urine shows wide $\mathrm{pH}$ variations and $\mathrm{pH}$ could cause a wide variation in xenobiotic distribution, it is mandatory to know urine $\mathrm{pH}$ and creatinine values. False positive and false negative results can condition the whole toxicological workup, and it should be kept in mind that every result must be critically evaluated by the experienced toxicologist.

\section{Saliva (Oral Fluid)}

Saliva, or oral fluid, has an electrolyte composition strictly related to blood and serum, so it is used as to a quick check, just to avoid venipuncture in roadside testing, specially for detecting Drug of Abuse. For oral fluid there is low, if any, place in post mortem toxicology. Its main role is, as nasal or oral swabs, for detecting a previous and recent exposure to an inhalant toxicant, or to a caustic substance.

\section{Vitreous Humor and Cerebrospinal Fluid}

Vitreous Humor (VH) is usually tested in Forensic Toxicology to detect a large number of xenobiotics. There are some interesting characteristics that give account of the reliability and usefulness of this kind of matrix, first of all it is relatively easy to obtain the sample, and the sample itself is less subject to bacterial contamination, so it is less prone to putrefactive processes. Secondly the environment is relatively poor of enzymes and proteins, so a drug is less subject to protein binding or enzymatic lysis [9-11]. However Vitreous Humor and Cerebrospinal Fluid (CSF) electrolyte composition is close to that of blood and serum and for this reason they could be useful alternative matrices. Many Authors have stressed the importance of alcohol detection and alcohol quantification in Vitreous Humor, which is a valuable alternative when the sample must be obtained from putrefied corpses, in air disasters or simply when femoral vein is not accessible. Alcohol in Vitreous Humor also could be an help when the toxicologist has to distinguish from ante mortem alcohol ingestion and alcohol post mortem production: because putrefactive processes are dilated in a "relatively protected environment" as eye chambers. Innovative chronic alcohol abuse markers could be tested in Vitreous Humor: Carbohydrate Deficient Transferrin (CDT) and Etilglucuronide (EtG) could widen our window of detection to define a background of chronic alcohol intake in an acute lethal alcohol intoxication. For many Authors the diagnosis of Digitalis intoxication relies mostly on digitalis detection in Vitreous Humor, and for this kind of lethal poisoning the Vitreous Humor can be defined as the Gold Standard matrix.

\section{Keratinized Tissues (Hair, Nails, Horny Layer of the Skin)}

The general term keratinized tissues should comprehend hair and nails (fingernails), but the matrix most often tested is hair. There are a plenty of papers about the supposed poisoning of Emperor Napoleon by arsenic and in spite of these also referenced studies, the only resulting data is the usefulness of hair analysis in chronic exposure to metal poisoning.

Hair is historically the most studied matrix for detecting poisons, and nowadays its role is essential to gain a retrospective information about drug abuse and poisoning, so hair analysis has gained the role of body of evidence by numerous Courts of Justice.

What gives account of the relevance of hair analysis is the real role of reservoir of this kind of matrix. This sample can provide a punctual anamnestic evaluation of drug or poison consumption, and this information has a wide detection window, mostly related to the overall length of the hair shaft analyzed. Drugs detected in hair comprehend: cocaine, benzoylecgonine, norcocaine, cocaethylene, morphine, codeine, oxycodone, hydrocodone, tetrahydrocannabinol, $\Delta 9$ - tetrahydrocannabinol and its major metabolites. This kind of analysis is also useful for a check of antiepileptic therapy in refractory epilepsy and in antipsychotic therapy [14-16].

There is no a best way to perform post mortem hair analysis, in our experience we suggest to have hair cut before the beginning of the autopsy, as to avoid further blood contamination. It is worthwhile the cut hair strands as close as possible to the scalp, possibly at the posterior vertex, where the rate of growth is constant. It is otherwise crucial to identify the scalp end as to correctly understand the rate of growth. This kind of sample is easy to collect and to storage and it be collected also from exhumed human bodies. Hair and finger nail could be a relevant specimen when heavy metal poisoning is suspected: specially for detecting arsenic, mercury or thallium. After hair sampling, there are other important phases as segmentation, washing in order to perform the screening test [17-20].

Immunochemical or immunometric screening tests are nowadays performed in every forensic laboratory, their main scope is to save time and to help the toxicologist to follow a correct diagnostic flow chart. As Guide Lines state every toxicological analysis must be performed by two different and independent methods, specially when there is a positive result. Confirmation tests should be performed by a chromatographic method coupled by mass spectrometry to improve specificity and sensibility. Segmental hair test is mandatory in rape or in suspected child abuse.

\section{Bile and Gastric Content}

The Bile is a reservoir for many drugs and toxicants, and for all the drugs which display hepatic metabolism and are subjected to entero-hepatic circulation. Heavy metals, benzodiazepines, paracetamol, cocaine, opiates could be found in bile, but nothing could be inferred about the exact timing of supposed intoxication [8-11]. 
The study of gastrointestinal contents is a crucial step because the oral route is a preferential route of administration of drugs and toxicants. The toxicologist should be aware of the uneven distribution of the material present, and it should be kept in mind the importance of relating the total amount of drug or poison to the total body mass. Another important item to remind is that it could be passive circulation from blood to stomach, so it is very difficult to state the lethal dose of the toxicant.

\section{Tissues Samples}

When there is no way to get blood, urine or other biological fluid the toxicological diagnosis can rely only to tissue analysis. Which tissue and how much tissue? It is impossible to answer to this question because every tissue can have its own peculiarity.

It is important to know the pharmacokinetic and pharmacodynamic properties of the suspected drug, which can affect the analysis: is the drug involved lipophilic and bound to the tissue to sample, what is its Distribution Volume? Moreover this kind of analyses is performed on exhumed or in putrefied bodies [12-15].

The Liver is a valuable specimen whenever blood is not available. Liver is usually suitable for detecting tricyclic antidepressants and neuroleptics, but it should be kept in mind that every drug subjected to entero-hepatic circulation can be found in liver tissue, like opioids and a great number of toxicants.

Because the Brain is the target and the site of action of lipophilic substances there is a rational in measuring drugs as Benzodiazepines, Antidepressants, cocaine and its metabolites. There is variability among the different anatomical regions of the brain, so the site of collection of samples must clearly defined between the pathologist and the toxicologist.

Bone Marrow is relatively protected from putrefactive process, is highly vascularized and it could be considered a real reservoir of drugs and toxicants. Analyses should be performed to test benzodiazepines, barbiturates and antidepressant, but in advanced stages of putrefaction it is impossible to define punctually the lethal dose, and because the loss of tissue it is only possible a qualitative result.

The main advantage of sampling Skeletal Muscle in post mortem setting is that skeletal muscle is present in large quantities and is less affect by putrefactive processes than other internal organs. Skeletal muscle is a reservoir and it is useful specially in anti-doping procedures.

Cardio-active drugs, as digitalis, anti-angina drugs, calcium entry blockers and other anti-arrhythmic drugs, antidepressants, opioids and heavy metals are all detectable in Cardiac Tissue. The information which emerges from this kind of complex analysis must be obviously related to circumstantial data provided by the pathologist.

All the drugs, toxicants, heavy metals and metabolites which are excreted by the Kidney are also found in Renal Tissue and sometimes, this tool could be considered as a positive control.
Also Adipose Tissue acts as reservoir for lipophilic substances, specially for cocaine, benzoylecgonine, amphetamine, metamphetamine and heavy metals [10-16].

\section{Insects}

Even if some toxicologists are skeptic about the usefulness of analysing insects, it should be stressed that insects may represent a useful source to research xenobiotics. The main goal is to determine toxicant concentration before death and to extrapolate these data to understand the causative role of toxicant itself. This kind of analysis is requested when the drug determinations should be performed on skeletonized or highly decomposed remains. According with Kintz et al. it is better to perform analysis on larvae instead of decomposed material. There are some controversies about the proper pre-analytical sample handling, because this condition could alter the result itself. However species as Coleoptera and Diptera are preferred because are first to colonize the corpse, the toxicologist should be aware of some limitations related to: different larval stages, different habit in searching for food, drug metabolism and different bioclimatic factors.

A relevant variable is weather itself: rain, temperature, photoperiod and humidity can influence factors as oviposition and the development of larvae and insects. In spite of these variables insect, or better, larval tissue analysis is a crucial help to have a qualitative result. Larval tissue analytic phases do not differ from solid tissue analysis, but sometimes in identifying properly the species of the insect to study it is better to rely on entomologist's experience.

Overall drug concentration relies on insect stage of development, and on the kind of tissue the larvae has fed. The xenobiotics detected are mostly heavy metals, barbiturates, but also in this contest the only inference possible is qualitative $[9,13,18]$.

\section{CONCLUSIONS}

What is considered the principal, easy and simple question in forensic toxicology could be otherwise the principal toxicologist's night-mare: had a toxicant or a drug a fundamental role to the death? The answer might be apparently simple, but really it is the most difficult challenge for the toxicologist and for the medical examiner too. Despite the progress and the increased sensibility and sensitivity in laboratory technology and laboratory methods, more often the whole interpretative process of results relies only on pathologist's and toxicologist's experience. Sample collecting, method optimizing, standard and mass spectra reviewing are only a minimal example of the challenges to face.

In our experience the only way to answer these questions is to elaborate a real Search Strategy in order to optimize laboratory resources and to elaborate a useful diagnostic flow chart. First of all is mandatory a full knowledge of circumstantial data and, in this context, what is the essential supposed role of quantitative data?

Actually it should be kept in mind that quantitative data are mostly derived from classical pharmacology concepts, 
that are largely invalid, because the pharmacokinetics and pharmacodynamics in living people are quite different from the same parameters in post-mortem.

To understand this condition we should be aware of post mortem circulation, wide $\mathrm{pH}$ variations, so called reservoir organs and early and late stages of decomposition. The correct approach should include the analysis of the afore mentioned circumstantial data, the thoroughly analysis of medical history and, most of all, the close relationship to the medical examiner.

A careful and complete sampling is mandatory, because it is the only way to obtain a lot of information as to complete the "puzzle" to solve the dilemma. So in our experience is better to sample more than less, so it is better to collect different tissue samples and body fluids so we are able to get more information, even if we are otherwise well aware that data results are to relate to pathologist's own experience and to clinical data [13-18].

If the so called quantitative approach could be prone to misinterpretations, which might be the correct approach? In our experience the best way is to avoid back calculations in defining the ante mortem drug concentrations, but it is better there is a useful dialogue between the toxicologist and the pathologist, or even best, for the toxicologist to actively assist to the autopsy, discussing and helping the pathologist to decide which kind of sample to collect.

The relevance of synergy between forensic pathologist and toxicologist emerges from the final discussion and evaluation of the case under study.

Some cases are clear cut for the pathologist because the cause and manner of death is straightforward from a pathological point of view, without the presence of significant quantities of substances able to exert a causal effect.

Other cases have no problems because the explanations reside in the work of the toxicologist, who finds substances, who explains everything, and the autopsy finding are negative.

Other cases are characterized by an explanation requiring the contribution of both pathologist and toxicologist for the presence of an interaction between pathologies of the patient and the presence of xenobiotics.

And finally there are the most difficult cases in which only a deep discussion between toxicologist and pathologist can explain some controversial points.

\section{CONFLICT OF INTEREST}

The authors confirm that this article content has no conflict of interest.

\section{ACKNOWLEDGEMENTS}

Declared none.

\section{REFERENCES}

[1] Brinkmann B. Harmonisation of medico-legal autopsies rules. Int J Legal Med 1999; 113: 1-14.

[2] Burton J, Rutty G Eds. The hospital autopsy, $2^{\text {nd }}$ ed. London: Arnold 2001; vol. 42: p. 126.

[3] Knight B. Forensic Pathology. $2^{\text {nd }}$ Ed. London: Arnold 1996; p. 533 .

[4] Madea B, Mußhoff F. Postmortem toxicology. Forensic Sci Int 2004; 142: 71-3.

[5] Shepherd R, Simpson's Forensic Medicine, $12^{\text {th }}$ ed, London: Arnold 2003; p. 34.

[6] Pelissier-Alicot AL, Gaultier JM, Champsaur P, Marquet P. Mechanism underlying post-mortem redistribution of drugs: a review. J Anal Toxicol 2003; 27: 53-44.

[7] Pounder DJ, Smith DRW. Postmortem diffusion of alcohol from the stomach. Am J Forensic Sci 1995; 16: 89-96.

[8] Pounder DY, Adams E, Fuke C, Langford AM. Site to site variability of post-mortem drug concentrations in liver and lung. J Forensic Sci 1996; 41: 927-32.

[9] Flanagan RJ, Connally G. Interpretation of analytical toxicology results in life and post-mortem. Toxicol Rev 2005; 24: 51-6.

[10] Leikin J, Watson W. Post-mortem toxicology: what the dead can and cannot tell us. J Clin Toxicol 2003; 41: 47-56.

[11] Richardson T. Pitfalls in forensic toxicology. Ann Clin Biochem 2000; 37: 20-44.

[12] Yarema MC, Becker CE. Key concepts in post-mortem drug redistribution. Clin Toxicol 2005; 43: 235-41.

[13] Harding-Pick D, Fryc O. Assessing death by poisoning: does the medical history help? Med Sci Law 1991; 31: 69-75.

[14] Prouty RW, Anderson WH. The forensic science implications of site and temporal influences on post-mortem blood drug concentrations. J Forensic Sci 1990; 35: 243-70.

[15] Jetter W. Postmortem biochemical changes. J Forensic Sci 1959; 4: 330-41.

[16] Mangin P, Kintz P. Variability of opiates concentrations in human hair according to their anatomical origin: head, axillary and pubic regions. Forensic Sci Int 1993; 63: 77-83.

[17] Seto Y. Determination of volatile substances in biological samples by headspace gas -chromatography. J Chromatogr A 1994; 674: 25-62.

[18] Liu JT, Hara K, Kashimura S, et al. Headspace solid-phase microextration and gas chromatographic - mass spectrometric screening for volatile hydrocarbons in blood. J Chromatogr B 2000; 748: 401-6.

[19] Baumgartner AM, Jones PF, Baumgartner W, Black CT. Radioimmunoassay of hair for determining opiate-abuse histories. J Nucl Med 1979; 20: 748-52.

[20] Cone E. Mechanism of drug incorporation into hair. Ther Drug Monit 1996; 18: 438-43. 